\title{
Which Style of Reasoning to Choose in the Face of Conflicting Information?*
}

\author{
Joke Meheus Christian Straßer Peter Verdée \\ Centre for Logic and Philosophy of Science \\ University of Ghent, Belgium \\ \{Joke.Meheus, Christian.Strasser, Peter.Verdee\}@UGent. be
}

\section{Introduction}

It is well-known that often different kinds of consequence relations are defined for reasoning from (possibly) inconsistent information. This holds true, for instance, for the consequence relations that are characterized in terms of maximal consistent subsets. The strong consequences are those formulas that follow by Classical Logic (CL) from every maximal consistent subset of the premises (in [18] these consequences are called inevitable consequences, elsewhere they are sometimes called universal consequences). The weak consequences (sometimes called existential consequences) follow by CL from some maximal consistent subset of the premises. The free consequences follow from the set of formulas that belong to every maximal consistent subset of the premises. ${ }^{1}$

Analogous distinctions can be found in default logics (and in logics that are defined in terms of them — such as some deontic logics, see for instance [8], and the signed systems from [4]). The sceptical consequences are analogous to the strong consequences (they follow from every extension) and the credulous consequences are analogous to the weak consequences (they follow from some extension). The prudent consequences from [4] are analogous to the free consequences (they follow from the set of formulas that belong to every extension). In Input/Output logics (see, for instance, [13]), we have the same distinction under the names "full meet constrained output" and "full join constrained output". Finally, we find this approach also in the context of argumentation

\footnotetext{
* Christian Straßer is a Postdoctoral Fellow of the Special Research Fund of Ghent University. Peter Verdée is a Postdoctoral Fellow of the Fund for Scientific Research - Flanders. Research of the first author was supported by subventions from Ghent University and from the Fund for Scientific Research - Flanders. The authors are indebted to the two referees for their valuable comments.

${ }^{1}$ In [3], the free consequences are defined as follows: where a free premise is one that does not belong to any minimal inconsistent subset of the premise set, the free consequences are all formulas that follow from the free premises. There, it has been shown that both definitions are equivalent since the premises that belong to every consistent subset are exactly the free premises.
} 
frameworks (see, for instance, [5]) where credulously accepted arguments are in some extensions of a given framework, and sceptically accepted arguments are in all extensions. ${ }^{2}$

Also for adaptive logics (which provide a unifying framework for all kinds of defeasible reasoning) different kinds of consequence relations are defined. This is done by varying the so-called adaptive strategy.

In recent years, many of the above consequence relations were characterized as adaptive logics. This not only offers a unified framework for these consequence relations, but also provides them with the same kind of semantics and the same kind of (dynamic) proof theory (and generic proofs for all important meta-theoretic properties). The strong consequences (and the sceptical consequences) as well as the free consequences (and the prudent consequences) can be reconstructed by means of the two 'standard' adaptive strategies (namely, Minimal Abnormality for strong/sceptical and Reliability for free/prudent). For the weak consequences, a new kind of adaptive strategy had to be designed (which is now called the Normal Selections strategy).

Up to now, adaptive logicians paid relatively little attention to the Normal Selections strategy (except for reconstructing and integrating existing consequence relations into the adaptive logic framework). Like some others, adaptive logicians have always been rather suspicious about the usefulness of the weak/credulous consequences.

The aim of the present paper is twofold. First, we want to address the concerns that some people have expressed with respect to the usefulness of the weak/credulous consequences. Next, we shall argue that, as a 'reasoning style', making weak/credulous inferences is sensible (for some application contexts), provided one has a (dynamic) proof theory for the corresponding consequence relation. Such a dynamic proof theory is exactly what adaptive logics offer. Finally, we shall illustrate all this by means of a very simple reconstruction of the free, strong, and weak consequences. We shall use the term Rescher-Manor consequences or RM-consequences when referring to all of them.

A characterization of the RM-consequence relations in terms of adaptive logics was first presented in [1]. That characterization relies on a paraconsistent logic and a translation of the premises in terms of both a classical and a paraconsistent negation. The present reconstruction proceeds entirely in terms of CL. ${ }^{3}$

The adaptive logics presented here are based on an 'extension' of CL, called $\mathbf{C L}_{\circ}$. The logic $\mathbf{C L}_{\circ}$ is obtained by adding the connective $\circ$ to the language schema of CL. As will become clear below, o functions as a 'block connective' in $\mathbf{C L}_{\circ}$ : from the truth or falsehood of $\circ A$ nothing follows about the truth or falsehood of $A$. Because of this, $\circ A$ functions as a 'block': it can be combined (by the usual formation rules) with other formulas, but not even replacement

\footnotetext{
${ }^{2}$ Our list is not exhaustive. We have a similar distinction in inheritance networks. However, there the sceptical consequences are presented as an alternative procedural approach to the one that results from intersecting the credulous extensions (see, for instance, [9] for details). In the context of both, inheritance networks and instantiated abstract argumentation, it is possible to distinguish between arguments that lie in the intersection of all extensions, and claims or formulas that can be derived from every extension (see, for instance, [9, 17]).

${ }^{3}$ For an introduction to adaptive logics, see, for instance [2].
} 
of equivalents is valid within the scope of $\circ$. For instance, from $\{\circ(p \wedge q)$, or $\}$, $\circ(p \wedge q) \wedge$ or is $\mathbf{C L}_{\circ}$-derivable, but $\circ(q \wedge p)$ is not.

The idea behind the reconstruction is extremely simple. First, the original premise set is translated by putting the unary connective $\circ$ in front of each premise. Next, the original premise set is restored 'as much as possible'. What this comes to is that in the adaptive $\operatorname{logics} A$ is "as much as possible" inferred from $\circ A$. It is precisely this property that allows for a very natural reconstruction of the RM-consequence relations. Depending on the adaptive strategy, one obtains a different interpretation of "as much as possible" (this will become clear below).

The adaptive logics will be presented in Sections 4-6, but first we address the question which style of reasoning is best suited for which context (Section 2 ) and the concerns regarding the weak/credulous consequences (Section 3).

\section{Different Reasoning Styles for Different Con- texts}

Ever since the idea originated to approach inconsistent theories in terms of 'extensions' of some kind (maximal consistent subsets or extensions of a default theory), there has been the question how the consequences should be defined in case there are multiple extensions.

In a recent book, Horty claims that this "question is vexed, and has not been adequately addressed" ([8, p. 35]). Like Horty, we shall not attempt to solve the problem here, but only point out a number of things.

We shall distinguish between four different options. The first one is the free/prudent option. This is one of the standard options in the RM-approach, but has been given relatively little attention outside that approach. The prudent consequence relation from [4] is the only analogue that we could find.

The free/prudent consequences are the 'innocent bystanders': they are in no way related to the inconsistencies, and hence, even in situations in which the inconsistencies have not yet been resolved, they can safely be accepted. Obviously, the set of free/prudent consequence is always consistent.

The latter also holds for the strong/sceptical consequences. Makinson, see [11, p. 38], also uses the term "conservative" for this option. It is typical of strong/sceptical reasoners that they refuse to draw conclusions in ambiguous situations (see, for instance, [15] and [21]). For instance, in the Nixon Diamond a sceptical reasoner will neither conclude that Nixon is a pacifist nor that he is not a pacifist.

The thirth and fourth options are the weak/credulous option and the choice option. For the first of these, Makinson also uses the terms "liberal" and "brave", see [11, p. 38]. It seems to us that these two categories are sometimes conflated in the literature and that at least part of the criticism against the weak/credulous option is related to this. We shall immediately come back to this.

The idea behind the weak/credulous option is to conclude as much as possible from an inconsistent theory (see, for instance, [21]). Thus, in the Nixon 
Diamond, one both concludes that Nixon is a pacifist and that he is not. According to the choice option, one simply chooses between different outcomes. For instance, one may decide to accept that Nixon is a pacifist, even when clear grounds are missing to give priority to this conclusion, rather than to its opposite.

As to the question which of these options is best suited for which reasoning context, there is no agreement. The opinions on the strong/sceptical option seem to be best in line with one another. Several authors point out that this option has some clear advantages: it leads to a consistent set of consequences, it is easy, and it is intuitively appealing (for instance, the idea that one should withhold belief in cases where one is dealing with conflicting information).

As for the choice option and the weak/credulous option, matters seem less clear. Some authors, such as Pollock, dismiss credulous reasoning as "just wrong". He defends this idea by means of the following example:

Suppose you have two friends, Smith and Jones, whom you regard as equally reliable. Smith approaches you in the hall and says, "It is raining outside." John then announces, "Don't believe him. It is a fine sunny day." If you have no other evidence regarding the weather, what should you believe? It seems obvious that you should withhold belief, believing neither that it is raining nor that it is not. If you were to announce, "I realize that I have no better reason for thinking that it is raining than for thinking that it is not, but I choose to believe that it is raining", no one would regard you as rational." ([15, p. 63])

In our view, this is not an example of credulous reasoning (where you keep an open mind for both options), but an example of the choice option (where, without good reasons, you just 'jump' to one of the conclusions). We would also agree that for this specific (epistemic) question ("Is it raining or not?"), the choice option does not seem justified.

Pollock continues by pointing out that credulous reasoning has sometimes been defended for practical reasoning, on the grounds that, in making practical decisions, it is sometimes better to act than not to act. With an example from Pollock: if a group wants to organise a picnic and the two possible sites are incomparable, then it seems rational to choose at random. However, Pollock immediately adds an example to show that at random choosing is not justified in every situation in which some practical decisions have to be made. In the words of Pollock:

If the agent is performing medical diagnosis and the evidence favoring two diseases is tied, we do not want the agent to decide randomly to treat the patient for one disease rather than the other. It could happen that the diseases are not serious if left untreated, but if the patient is treated for the wrong disease, that treatment will gravely exacerbate his condition. In such a case we want the agent to reserve judgment on the matter and not proceed blindly. $([15$, p. 63$])$ 
In our view, Pollock's three examples differ in important respects. The first example concerns a purely epistemic context (the agent wants an answer to the question whether or not it is raining). The second example seems to concern an application context that is mainly (or even purely) practical (and in which, moreover, no harm can be done by acting rather than not acting). The third example seems to involve both epistemic aspects and practical aspects. Treating the patient in a justified way also means that one should at least try to obtain the best possible insight in the underlying causes. This in turn may require that one investigates the different alternatives and their different outcomes. In our view, this purpose is best served by the weak/credulous option (for which we ourselves prefer the term "explorative").

If we understand him well, the idea that some reasoning contexts involve both epistemic aspects and practical aspects can also be found in Prakken's [16]. Prakken uses the following example. Some university lecturer, John, wants to finish a paper by Friday, but also promised to give a lecture in some remote town Faraway. As far as John believes, there are only two ways to get to Faraway: by car or by bus. In either case, he will not be able to finish the paper by Friday (he always becomes sick when reading or writing on a bus). So, he is dealing with a conflict between a desire and a promise. As John sees no reason to prefer either his desire to his promise or his promise to his desire, it is, according to Prakken, rational for John to choose randomly whether he will fulfill his desire or keep his promise. Again according to Prakken, this reasoning should be formalized as credulous reasoning.

Also here, our analysis would be that we are dealing with the choice option rather than with credulous reasoning. For us, this example is similar to the picnic example. There are two (incompatible) goals, there seems to be no rational ground for choosing between them, and realizing one of the goals is better than doing nothing. So, in this case (if the possible actions are indeed indifferent in all respects), we have no problem with the choice option.

Prakken then goes on to elaborate on the example. Suppose that John's friend Bob informs him that there is also a train to Faraway, which would give him the option to work on the paper and still give the lecture. However, John's other friend Mary warns him that there will be a strike on Friday, and that there will be no trains to Faraway, which is denied by Bob. So, before John sets the goal to take the train, he should first find out whether or not there will be a train. If John has no reason to trust one friend more than the other, he should, according to Prakken, reason in a sceptical way, and not act on the credulous belief that there will be a train to Faraway on Friday. Prakken draws the following conclusion from the example:

The kind of rationality that is assumed here is that a rational agent should map out all credulously acceptable action alternatives that have sceptically acceptable epistemic support and then make a choice between them. ([16, p. 312])

A similar defence for the use of credulous reasoning in mapping out the different alternatives can also be found at the end of Prakken's paper: 
[I]n some contexts all reasoning can best be credulous while in other contexts epistemic reasoning can better be sceptical (it makes less sense to make all reasoning sceptical, since practical reasoning inevitably involves choice and a logic for such reasoning should reveal the alternatives). ([16, p. 321])

Also in [21, p. 478] a similar advantage is pointed out for the credulous approach: this approach enables one to generate all possible extensions, after which they can be examined for interesting properties.

Let us now try to summarize our own position on this matter in three points. The first is that the choice option and the credulous option should not be identified. In our view, much of the criticism against the credulous option is precisely related to this conflation.

Our second point is that credulous reasoning (or explorative reasoning as we prefer to call it), can be useful in many situations where one is dealing with conflicting information. One of the reasons for this is that, in real life examples, it is seldom the case that different options are equal in all respects and that we decide about them by flipping a coin. In Prakken's example, for instance, going to Faraway may be a rare chance to meet with some colleagues and to talk about a joint project. And, normally several other interests and consequences of our actions will be involved. The most rational way to proceed in cases like this is to weigh the different pros and cons and to try to design some priority ordering. However, before one is able to do this, one needs to know what the different options and their expected outcomes are. In order to find that out, one will have to reason from the information one has. Explorative reasoning seems to be best suited here.

Our third point is that it is not evident to link the sceptical/credulous distinction to the epistemic/practical distinction. As far as we see it, explorative reasoning occurs in purely epistemic contexts, in purely practical contexts and also in 'mixed' contexts. We also believe that all kinds of pragmatic factors may influence one's reasoning strategy. ${ }^{4}$ Let us try to illustrate this with some examples.

Suppose you are a pilot in a plane that is in trouble (for instance, you obtain conflicting information from some of your instruments). If you are not already at the stage that immediate actions are needed to prevent the plane from crashing, it may be wise to do some really quick thinking and to explore the different possibilities before acting. However, if you are unsuccessful in ruling out the competing explanations (in such a way that only one remains), you will at some point have to switch to the choice mode. (We assume here that not acting definitely will lead to a crash.) This is typically a context where one has a complex interaction between epistemic aspects and practical aspects (in order to decide on the best possible actions, you need good hypotheses on

\footnotetext{
${ }^{4}$ In the context of inheritance networks Horty seems to point out to one particular pragmatic consideration that sometimes plays a role. According to Horty, the credulous "option seems appropriate for situations in which the value of drawing conclusions is high relative to the costs involved if some of those conclusions turn out not to be correct", while "when the cost of error rises [...] an ideal reasoner's conclusion set [...] should be determined, somehow, by the intersection of these extensions." ([9, p. 123]).
} 
what causes the conflicting readings) and where also other factors come into play (for instance, that at some point you are running out of time).

Compare this with investigators who have to find out what caused a plane to crash. In this case, all that matters is that one finds the true (or most likely) cause so that similar crashes can be avoided in the future. In the meantime, it is harmless that different people start exploring different paths (or even the same person at different moments in time). A similar situation can be found when scientists are trying to find an explanation for some hitherto unexplained phenomenon. In these cases, there may also be some time pressure (reports and papers are often due for a certain date), but it will not have the same impact as in the example from the previous paragraph. What matters here is that one finds the true (or most likely) explanation and this is best served by a period of exploratory reasoning. So, what we seem to have here are examples of epistemic contexts, where, at least for part of the reasoning involved, one best opts for an explorative reasoning style.

As was pointed out in the introduction, the same three kinds of consequence relations keep reappearing in the literature. By lack of better terms (and so as not to associate ourselves with one of the existing paradigms), we choose "safe", "cautious" and "explorative" for the three reasoning styles.

In the case of the RM-consequence relations, the safe consequences are the free consequences. As they are not related to the inconsistencies, they can safely be relied upon. For instance, no matter what causes the plane to crash, it is safe to instruct the passengers about the appropriate safety instructions.

The strong consequences correspond to the cautious reasoning style. Suppose that our university professor at some point decides to give the lecture, but that he is still uncertain whether there will be a strike or not. Suppose moreover that he decides that he will go by bus, if there is a strike. As it happens, there is only one bus, arriving at 1pm in Faraway, and only one train, arriving at 10am. At this point, he may want to inform his host that he will in any case be coming and that he will arrive either at $10 \mathrm{am}$ or at $1 \mathrm{pm}$ (so that his host can start making the necessary arrangements for the lecture, taking into account both scenarios until he has a definite answer). ${ }^{5}$

What we dub the "explorative style" is typical of situations where there is no time pressure and where there is no immediate need to take precautionary measures, but where, for instance, one tries to find out what is the truth of the matter. This is also why we choose a term that does not have the negative connotations that "weak" and "credulous" have.

\footnotetext{
${ }^{5}$ The conclusion that he will come either at $10 \mathrm{am}$ or $1 \mathrm{pm}$ is a so-called floating conclusion. A floating conclusion can be reached by means of two conflicting arguments. In this case, we have the argument that leads to the claim that he arrives at $10 \mathrm{am}$ and the conflicting argument that leads to the claim that he arrives at $1 \mathrm{pm}$. From both we can derive that he arrives either at $10 \mathrm{am}$ or at $1 \mathrm{pm}$. There is a vivid debate concerning the question whether and when floating conclusions should be accepted (see [7, 10, 12]). In [19] the notion of floating conclusions has been linked to the strategies of adaptive logics: according to the minimal abnormality strategy they are accepted, while according to the reliability strategy they are rejected.
} 


\section{Weak Consequences versus Explorative Infer- ences}

In the previous section, we tried to make a case that, in some situations, reasoning in accordance with the weak/credulous consequences can by useful. Still, many readers may have doubts. In this section, we shall consider some of the concerns that have been raised against the motivation and the rational use of the weak consequences.

If one considers the set of weak consequences, it seems unavoidable to conclude that, in most cases, it is just a terrible loss of information. Let us give an example. Suppose one starts from the following premises:

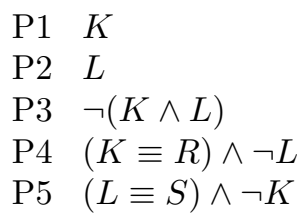

In this case, there are four different maximal consistent subsets:

$$
\begin{array}{ll}
\text { MCS1 } & \{\neg(K \wedge L), K,(K \equiv R) \wedge \neg L\} \\
\text { MCS2 } & \{\neg(K \wedge L), L,(L \equiv S) \wedge \neg K\} \\
\text { MCS3 } & \{K, L\} \\
\text { MCS4 } & \{\neg(K \wedge L),(K \equiv R) \wedge \neg L,(L \equiv S) \wedge \neg K\}
\end{array}
$$

Hence, the weak consequences of the premises are the members of the following set:

$\Delta=C n(\{K, \neg L, R\}) \cup C n(\{\neg K, L, S\}) \cup C n(\{K, L\}) \cup C n(\{\neg K, \neg L, \neg R, \neg S\})$

This set of consequences is not closed under any reasonable logic, and so is definitely not useful as a stand-alone theory. But does it filter useful information out of the inconsistent premises? Well, arguably it does not. The inconsistencies have spread so widely, that all implicational information from the original premises seems to be lost. For instance, $K \supset R$, which follows from the fourth premise, is a member of $\Delta$, but so are $K \supset \neg R, R \supset K$ and $R \supset \neg K$.

One could see the set of weak consequences as a collection of suggestions for hypotheses for further research. However, as the set is per definition infinite, one would need an extra filtering mechanism for every practical purpose. Moreover, if one finds out that one of the consequences does not hold true, there is no way to use this new result for further reasoning when starting from the members of $\Delta$. Suppose, for example, one finds out convincingly that $L$ is not the case. We may conclude that we should give up on $L$. Should we then also give up on $L \vee R$ or $S \vee L$ ? The set of weak consequences by itself does not give us any clue on how to proceed in such cases. Hence, if one is concerned with extracting useful information from inconsistent premises, the weak consequences do not seem the best option around. 
But suppose that you find yourself in the following situation. You are a member of some committee that has to select two candidates for two open positions. Suppose further that after some discussion the committee agreed to narrow down the number of potential candidates up to four: Kaleb, Lauren, Rose and Stephen. Now let $K$ stand for "Kaleb should get one of the positions", and analogously for the other letters occurring in the premises (and the corresponding names). You yourself have a clear preference for hiring both Rose and Stephen. However, your favourite strategy in such cases is to listen first to the arguments of the others and to try to figure out in which direction the discussion is going, before you start entering the debate yourself.

It is easily imaginable that you are confronted with the following situation. Two participants in the debate, P1 and P2, make a strong case in favour of, respectively, Kaleb and Lauren. A third participant P3 does not (as yet) express any clear preferences about Rose and Stephen, but definitely does not want that both Kaleb and Lauren are selected (because, for instance, P3 suspects that their characters are incompatible and that they might fight all the time when they become members of the same group). A fourth participant $\mathrm{P} 4$ definitely does not want Lauren to get the job, but P4 also makes it clear that Kaleb should be hired if and only if also Rose is hired (for instance, P4 agrees with $\mathrm{P} 1$ that $\mathrm{K}$ would be a very good candidate, but also believes that Kaleb lacks certain skills and that, of the three others, Rose is the most compatible with him). Finally, P5 does not like Kaleb, but also argues that Lauren should be hired if and only if Stephen is hired.

If you yourself want to make a strong case in favour of hiring both Rose and Stephen, it might be wise to try to figure out first which participants have compatible views and which have not (for instance, because you know from experience that, in some cases, "compatible subgroups" start supporting each other in an attempt to direct the discussion in a certain way). But, in order to figure this out, you will have to reason from their statements. What this comes to is that you will have to make inferences from the premises, in such a way that you not only avoid the derivation of trivial conclusions, but also that you are able to detect, for instance, which statements are jointly compatible.

In Section 7, we shall show that a dynamic proof theory is a useful tool to explicate this kind of explorative reasoning. Hopefully, it will also become clear that knowing about this kind of proofs and how they work may make one a better reasoner and a better debater. But, first we have to present the adaptive logics.

\section{Adaptive Logics for the RM-Consequences}

Although the original RM-consequence relations were defined in [18] at the propositional level, we shall extend them to the predicative level. Let $\operatorname{MCS}(\Gamma)$ stand for the set of maximal consistent subsets of $\Gamma$ and let Free $(\Gamma)$ stand for $\{A \mid A \in \Delta$, for each $\Delta \in \operatorname{MCS}(\Gamma)\}$. The three consequence relations are defined by:

Definition $1 \Gamma \vdash_{\text {Free }} A$ iff $F r e e(\Gamma) \vdash_{\mathbf{C L}} A$. 
Definition $2 \Gamma \vdash_{\text {Strong }} A$ iff $\Delta \vdash_{\mathrm{CL}} A$ for all $\Delta \in \operatorname{MCS}(\Gamma)$.

Definition $3 \Gamma \vdash_{\text {Weak }} A$ iff $\Delta \vdash_{\mathbf{C L}} A$ for some $\Delta \in \operatorname{MCS}(\Gamma)$.

The adaptive logics that we present here are based on $\mathbf{C L}_{\circ}$ and will be called $\mathbf{C L}_{\circ}^{r}$ (for the Reliability strategy), $\mathbf{C L}_{\circ}^{m}$ (for the Minimal Abnormality strategy) and $\mathbf{C L}_{\circ}^{n}$ (for the Normal Selections strategy). We shall use $\mathbf{C L}_{\circ}^{x}$ when referring to properties that are common to all three logics.

As all adaptive logics, the logics $\mathbf{C} \mathbf{L}_{\circ}^{x}$ are characterized by a triple: (i) a lower limit logic LLL, (ii) a set of abnormalities $\Omega$, characterized by a logical form, and (iii) an adaptive strategy.

What all adaptive logics have in common is that they interpret a given set of premises "as normal as possible". What "normal" means depends on the abnormalities. ${ }^{6}$ When a number of conditions are fulfilled, the adaptive logic is in standard format. For adaptive logics in standard format, there are generic proofs for all central meta-theoretic properties (see [1]). The conditions for an adaptive logic to be in standard format are: (i) the LLL has to be reflexive, transitive, monotonic, and compact and (ii) the strategy should be Reliability or Minimal Abnormality. The logics $\mathbf{C} \mathbf{L}_{\circ}^{r}$ and $\mathbf{C L}_{\circ}^{m}$ are in standard format, the $\operatorname{logic} \mathbf{C L}_{\circ}^{n}$ is not. However, as is shown in [20], every adaptive logic that has Normal Selections as its strategy is characterized (under a certain translation) by an adaptive logic in standard format.

The lower limit logic of all three systems is $\mathbf{C L}_{\circ}$. We shall use $\mathcal{L}$ to refer to the standard predicative language schema, and $\mathcal{L}_{\circ}$ to refer to $\mathcal{L}$ extended with $\circ ; \mathcal{W}$ and $\mathcal{W} \circ$ will refer to their respective sets of wffs. The members of $\mathcal{W} \circ$ are obtained by the usual formation rules, except that $(\forall \alpha) A$ and $(\exists \alpha) A$ are only well-formed if $A \in \mathcal{W}$ and that iterated occurrences of the connective $\circ$ are not allowed. We shall use $\Gamma^{\circ}$ to refer to $\{\circ A \mid A \in \Gamma\}$.

The axiomatization of $\mathbf{C} \mathbf{L}_{\circ}$ is as for $\mathbf{C L}$. The semantics is obtained in a straightforward way from that for CL. Let $M=\langle D, v\rangle$ be a standard CLmodel, with $D$ the domain and $v$ the assignment function. The assignment $v$ of a $\mathbf{C} \mathbf{L}_{\circ}$-model is exactly like an assignment of a $\mathbf{C L}$-model, except that the latter is extended in such a way that also formulas of the form $\circ A$ get a value in $\{0,1\}$. For formulas of the form $\circ A$, the valuation function $v_{M}$ determined by a model $M$ simply follows the assignment function. So, we add the clause:

$$
v_{M}(\circ A)=1 \text { iff } v(\circ A)=1 .
$$

All other clauses for the valuation function are as for CL.

We now turn to the set of abnormalities for our three logics and to the three strategies. Consider the premise set $\Gamma_{1}=\{p, q, \neg p \vee \neg q, t \wedge \neg t\}$. In order to find out what the free, strong and weak consequences of $\Gamma_{1}$ are, we translate the original premise set to $\Gamma_{1}^{\circ}=\{\circ p, \circ q, \circ(\neg p \vee \neg q), \circ(t \wedge \neg t)\}$. The question is now which premises of the form $\circ A$ should not be interpreted as $A$. This is obviously the case for $\circ(t \wedge \neg t)$. The reason is that $\neg(t \wedge \neg t)$ follows by $\mathbf{C L}_{\circ}$

\footnotetext{
${ }^{6}$ Note that "normality" is used here as a technical term. It does not refer to formulas that are considered false according to some standard of 'good reasoning' (CL, for instance), but to formulas that may overrule inferences that are, in some application context, considered as desirable but defeasible.
} 
from $\Gamma_{1}^{\circ}$. This gives us immediately an idea of the kind of abnormality that we are looking for. Abnormalities are formulas that overrule certain defeasible inferences. In this case, we want to interpret $\circ A$ as much as possible as $A$, but we want to invalidate this inference when $\circ A \wedge \neg A$ is derivable by $\mathbf{C L}_{\circ}$. For all three logics, abnormalities will be formulas of the form $\circ A \wedge \neg A$. So, the set of abnormalities $\Omega$ of the systems $\mathbf{C L}_{\circ}^{x}$ is $\{\circ A \wedge \neg A \mid A \in \mathcal{W}\}$.

From what was said above, it may seem that in adaptive logics, abnormalities are assumed to be false unless and until they are derivable by the LLL, in our case $\mathbf{C L}_{\circ}$. There is, however, a small complication. Consider, for instance, $\circ p$. Although $\circ p \wedge \neg p$ is not $\mathbf{C L}_{\circ}$-derivable from $\Gamma_{1}^{\circ}$, the disjunction $(\circ p \wedge \neg p) \vee(\circ q \wedge \neg q) \vee(\circ(\neg p \vee \neg q) \wedge \neg(\neg p \vee \neg q))$ is derivable. What this shows is that $p, q$ and $\neg p \vee \neg q$ are jointly incompatible. It is here that the adaptive strategy comes in. In cases where a disjunction of abnormalities is derivable by the LLL, but the separate disjuncts are not, the phrase "interpreting the premises as normal as possible" becomes ambiguous. The adaptive strategy is meant to disambiguate this phrase. What the three strategies come to is most easily introduced by means of the semantics, to which we now turn.

\section{The Semantics}

For adaptive logics in standard format, the semantics is obtained by selecting a subset of the models of the LLL that verify $\Gamma$. Intuitively, those models are selected that, in view of the adaptive strategy, are as normal as possible. Note especially that the intended selection can only be defined by referring to some set of premises. Hence, it does not make sense to say that some model of the LLL is an adaptive model. It only makes sense to say that it is an adaptive model of the set of premises at issue.

For adaptive logics that are based on the Normal Selections strategy, the selection requires a bit more explanation, but, as we shall see, is also quite intuitive.

Let us start with some notations and definitions that we need to explain the semantics of $\mathbf{C L}_{\circ}^{r}$ and $\mathbf{C} \mathbf{L}_{\circ}^{m}$. We shall use the term Dab-formula to refer to a disjunction of abnormalities. In any subsequent expression of the form $\operatorname{Dab}(\Delta)$, $\Delta$ is a finite subset of $\Omega$ and $\operatorname{Dab}(\Delta)$ is a disjunction of the members of $\Delta$-in practice we shall identify $\operatorname{Dab}(\Delta)$ with every disjunction of the members of $\Delta$.

The $D a b$-formulas that are derivable by the LLL from the set of premises $\Gamma$ are called the $D a b$-consequences of $\Gamma . \operatorname{Dab}(\Delta)$ is called a minimal Dabconsequence of $\Gamma$ iff there is no $\Delta^{\prime} \subset \Delta$ such that $\operatorname{Dab}\left(\Delta^{\prime}\right)$ is a $D a b$-consequence of $\Gamma$.

Where $M$ is a model of the LLL, the abnormal part of $M$ is denoted by $A b(M)$ and consists of all the abnormalities that are verified by $M$. Where $\operatorname{Dab}\left(\Delta_{1}\right), \operatorname{Dab}\left(\Delta_{2}\right), \ldots$ are the minimal $D a b$-consequences of $\Gamma$, the set $U(\Gamma)=$ $\Delta_{1} \cup \Delta_{2} \cup \ldots$ stands for the set of formulas that are unreliable with respect to $\Gamma$.

For $\mathbf{C} \mathbf{L}_{\circ}^{r}$, the semantic selection is determined by the following definitions:

Definition $4 A \mathbf{C L}_{\circ}$-model $M$ of $\Gamma$ is reliable iff $A b(M) \subseteq U(\Gamma)$. 
Definition $5 \Gamma \Vdash_{\mathbf{C L}_{\circ}^{r}}$ A iff $A$ is verified by all reliable models of $\Gamma$.

For the semantics of $\mathbf{C L}_{\circ}^{m}$, we need the following definitions:

Definition 6 A $\mathbf{C L}_{\circ}$-model of $\Gamma$ is minimally abnormal iff there is no $\mathbf{C L}_{\circ}$ model $M^{\prime}$ of $\Gamma$ such that $A b\left(M^{\prime}\right) \subset A b(M)$.

Definition $\mathbf{7} \Gamma \Vdash_{\mathbf{C L}_{\circ} m} A$ iff $A$ is verified by all minimally abnormal models of $\Gamma$.

Let us now turn to the semantics of $\mathbf{C L}_{\circ}^{n}$, which is in a sense 'derived' from the Minimal Abnormality strategy. In order to make this clear, we shall rely on some further conventions and definitions.

A choice set of $\Sigma=\left\{\Delta_{1}, \Delta_{2}, \ldots\right\}$ is a set that contains an element out of each member of $\Sigma$. A minimal choice set of $\Sigma$ is a choice set of $\Sigma$ of which no proper subset is a choice set of $\Sigma$. Where $\operatorname{Dab}\left(\Delta_{1}\right), \operatorname{Dab}\left(\Delta_{2}\right), \ldots$ are the minimal $D a b$-consequences of $\Gamma, \Phi(\Gamma)$ is the set of minimal choice sets of $\left\{\Delta_{1}\right.$, $\left.\Delta_{2}, \ldots\right\}$.

As was shown in [1], for every $\phi \in \Phi(\Gamma)$, there is a minimally abnormal model $M$ such that $M \models \Gamma$ and $A b(M)=\phi$, and vice versa. Evidently, this does not exclude that there are LLL-models $M$ and $M^{\prime}$ for which $A b(M)=$ $A b\left(M^{\prime}\right)=\phi$. This raises the question which formulas are verified by all LLLmodels that share the same (minimally) abnormal part. Here are the precise definitions for the semantics of $\mathbf{C L}_{\circ}^{n}$ :

Definition 8 A set $\mathcal{M}$ of $\mathbf{C L}_{\circ}$-models of $\Gamma$ is a normal selection iff, for some $\phi \in \Phi(\Gamma), \mathcal{M}=\{M \mid M \models \Gamma ; A b(M)=\phi\}$.

Definition $9 \Gamma^{\circ} \Vdash_{\mathbf{C L}_{\circ}^{n}} A$ iff $A$ is verified by every model of a normal selection of $\mathbf{C L}_{\circ}^{m}$ of $\Gamma^{\circ}$.

\section{The Dynamic Proof Theory}

The proof theory of adaptive logics is characterized by three rules (a premise rule PREM, an unconditional rule RU, and a conditional rule RC) and a marking definition. The rules are the same for all logics, the marking definition (which is determined by the adaptive strategy) is different.

The proofs themselves look like those of any other logic, except that every line has a condition attached to it. As we shall see, the condition is introduced by the rules and the marking definition acts upon it: whether a line is marked or not depends on its condition. Formulas that occur on marked lines are no longer considered as derived in the proof.

Where $\Gamma$ is the set of premises, the inference rules for the logics $\mathbf{C L}_{\circ}^{x}$ are given by: 
PREM If $A \in \Gamma$ :

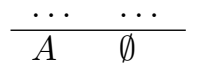

RU

If $A_{1}, \ldots, A_{n} \vdash_{\mathbf{C L}_{\circ}} B$ :

$A_{1} \quad \Delta_{1}$

... $\quad \cdots$

$\begin{array}{ll}\cdots & \cdots \\ A_{n} & \Delta_{n}\end{array}$

$B \quad \Delta_{1} \cup \ldots \cup \Delta_{n}$

$\mathrm{RC}$

$$
\begin{aligned}
& \text { If } A_{1}, \ldots, A_{n} \vdash_{\mathbf{C L}_{\circ}} B \vee \operatorname{Dab}(\Theta) \quad A_{1} \quad \Delta_{1} \\
& \text {... } \ldots \\
& \begin{array}{ll}
A_{n} & \Delta_{n} \\
\hline B & \Delta_{1} \cup \ldots \cup \Delta_{n} \cup \Theta
\end{array}
\end{aligned}
$$

The premise rule PREM simply states that, at any line of a proof, a premise may be introduced on the empty condition. What the unconditional rule RU comes to is that whenever $A_{1}, \ldots, A_{n} \vdash_{\mathbf{C L}}, B$ and $A_{1}, \ldots, A_{n}$ occur in the proof on the conditions $\Delta_{1}, \ldots, \Delta_{n}$, then $B$ may be added to the proof on the condition $\Delta_{1} \cup \ldots \cup \Delta_{n}$. The conditional rule $\mathrm{RC}$ is analogous, except that here a new condition is introduced.

Before we turn to the marking definitions, let us illustrate the inference rules by means of the example. We shall use $! A$ to refer to $(\circ A \wedge \neg A)$. Suppose that we are interested in finding out what the free, strong and weak consequences are of $\Gamma_{2}=\{p, q, \neg p \vee \neg q, \neg p \vee r, \neg q \vee s, t \wedge \neg t\}$ and that we start a $\mathbf{C L}_{\circ}^{x}$-proof by first entering the premises of the 'translated' premise set:

$\begin{array}{llll}1 & \circ p & \text { PREM } & \emptyset \\ 2 & \circ q & \text { PREM } & \emptyset \\ 3 & \circ(\neg p \vee \neg q) & \text { PREM } & \emptyset \\ 4 & \circ(\neg p \vee r) & \text { PREM } & \emptyset \\ 5 & \circ(\neg q \vee s) & \text { PREM } & \emptyset \\ 6 & \circ(t \wedge \neg t) & \text { PREM } & \emptyset\end{array}$

Suppose that we would now continue the proof as follows: ${ }^{7}$

$\begin{array}{llll}7 & p & 1 ; \mathrm{RC} & \{! p\} \\ 8 & q & 2 ; \mathrm{RC} & \{! q\} \\ 9 & \neg p \vee \neg q & 3 ; \mathrm{RC} & \{!(\neg p \vee \neg q)\} \\ 10 \neg p \vee r & 4 ; \mathrm{RC} & \{!(\neg p \vee r)\} \\ 11 \neg q \vee s & 5 ; \mathrm{RC} & \{!(\neg q \vee s)\} \\ 12 t \wedge \neg t & 6 ; \mathrm{RC} & \{!(t \wedge \neg t)\}\end{array}$

The unconditional rule now allows us to add, for instance, also the following lines:
$13 p \wedge q$
7,$8 ; \mathrm{RU} \quad\{! p, ! q\}$
$14(p \wedge q) \wedge(\neg p \vee \neg q)$
9,$13 ; \mathrm{RU} \quad\{! p, ! q, !(\neg p \vee \neg q)\}$

\footnotetext{
${ }^{7}$ Remark that the applications of the RC-rule on lines $7-12$ are justified in view of the fact that $\circ A \vdash_{\mathbf{C L}_{\circ}} A \vee ! A$.
} 


$\begin{array}{llll}15 & r & 7,10 ; \mathrm{RU} & \{! p, !(\neg p \vee r)\} \\ 16 & s & 8,11 ; \mathrm{RU} & \{! q, !(\neg q \vee s)\} \\ 17 & r \vee s & 15 ; \mathrm{RU} & \{! p, !(\neg p \vee r)\} \\ 18 & r \vee s & 16 ; \mathrm{RU} & \{! q, !(\neg q \vee s)\}\end{array}$

At this point some readers might be worried that now some plain contradictions are derived in the proof. However, as we shall immediately see, lines 12 and 14 are marked in the proof, according to all three strategies, as soon as the relevant disjunctions of abnormalities are derived. Note that the same formula, namely $r \vee s$ has been derived twice in the proof, but on a different condition. We shall immediately see that, in adaptive proofs, deriving the same formula on a different condition may make a difference for the marking.

We now set out to present the marking definitions. $\operatorname{Dab}(\Delta)$ is a minimal $D a b$-formula at stage $s$ of an adaptive proof iff $\operatorname{Dab}(\Delta)$ has been derived at that stage on the condition $\emptyset$ whereas there is no $\Delta^{\prime} \subset \Delta$ for which $\operatorname{Dab}\left(\Delta^{\prime}\right)$ has been derived on the condition $\emptyset$. Consider a proof from $\Gamma$ at stage $s$ and let $\operatorname{Dab}\left(\Delta_{1}\right), \operatorname{Dab}\left(\Delta_{2}\right) \ldots$ be the minimal $D a b$-formulas at that stage. Let $U_{s}(\Gamma)=\Delta_{1} \cup \Delta_{2} \ldots$ and define $\Phi_{s}(\Gamma)$ as the set of minimal choice sets of $\left\{\Delta_{1}, \Delta_{2} \ldots\right\}$.

Definition 10 Marking for Reliability: A line is marked at stage $s$ iff, where $\Delta$ is its condition, $\Delta \cap U_{s}(\Gamma) \neq \emptyset$.

Definition 11 Marking for Minimal Abnormality: A line on which $A$ is derived on the condition $\Delta$ is marked at stage $s$ iff $(i)$ there is no $\phi \in \Phi_{s}(\Gamma)$ such that $\phi \cap \Delta=\emptyset$, or, for some $\phi \in \Phi_{s}(\Gamma)$, there is no line on which $A$ is derived on a condition $\Theta$ for which $\phi \cap \Theta=\emptyset$.

This reads more easily: where $A$ is derived on the condition $\Delta$ on line $l$, line $l$ is unmarked at stage $s$ iff (i) there is a $\phi \in \Phi_{s}(\Gamma)$ for which $\phi \cap \Delta=\emptyset$ and (ii) for every $\phi \in \Phi_{s}(\Gamma)$, there is a line at which $A$ is derived on a condition $\Theta$ for which $\phi \cap \Theta=\emptyset$.

Definition 12 Marking for Normal Selections: Line l is marked at stage s iff, where $\Delta$ is its condition, $\operatorname{Dab}(\Delta)$ has been derived on the condition $\emptyset$ at stage $s$.

If, at stage $s$ of a proof from $\Gamma$, a formula $A$ occurs on a line that is not marked, then $A$ is said to be derived from $\Gamma$ at that stage of the proof. Whenever a line is added to the proof, lines that were previously unmarked may be marked and, except for the Normal Selections strategy, also vice versa. However, the notion of final derivability is independent of the way in which the proof proceeds:

Definition $13 A$ is finally derived in a proof from $\Gamma$ on line $i$ of a proof at finite stage $s$ iff (i) $A$ is the second element of line $i$, (ii) line $i$ is not marked at stage $s$, and (iii) every extension of the proof in which line $i$ is marked may be further extended in such a way that line $i$ is unmarked. 
As may be expected, the derivability relation is defined with respect to final derivability:

Definition $14 \Gamma \vdash_{\mathbf{C L}_{\circ}^{x}} A$ ( $A$ is finally derivable from $\Gamma$ ) iff $A$ is finally derived in an $\mathbf{C L}_{\circ}^{x}$-proof from $\Gamma$.

Let us return to the proof we started above to illustrate the marking definitions. This is what the proof looks like, for the different strategies, once the two (minimal) disjunctions of abnormalities that are derivable from the premises have been added to the proof - we do not repeat lines 1-6. Notations such as $\mathrm{R}^{19}$ indicate that a line is marked, on the basis of the Reliability strategy, in view of line 19 .

$\begin{array}{llll}7 & p & 1 ; \mathrm{RC} & \{! p\} \mathrm{R}^{20}, \mathrm{M}^{20} \\ 8 & q & 2 ; \mathrm{RC} & \{! q\} \mathrm{R}^{20}, \mathrm{M}^{20} \\ 9 & \neg p \vee \neg q & 3 ; \mathrm{RC} & \{!(\neg p \vee \neg q)\} \mathrm{R}^{20}, \mathrm{M}^{20} \\ 10 \neg p \vee r & 4 ; \mathrm{RC} & \{!(\neg p \vee r)\} \\ 11 \neg q \vee s & 5 ; \mathrm{RC} & \{!(\neg q \vee s)\} \\ 12 t \wedge \neg t & 6 ; \mathrm{RC} & \{!(t \wedge \neg t)\} \mathrm{R}^{19}, \mathrm{M}^{19}, \mathrm{~N}^{19} \\ 13 p \wedge q & 7,8 ; \mathrm{RU} & \{! p, ! q\} \mathrm{R}^{20}, \mathrm{M}^{20} \\ 14(p \wedge q) \wedge(\neg p \vee \neg q) & 9,13 ; \mathrm{RU} & \{! p, ! q !(\neg p \vee \neg q)\} \mathrm{R}^{20}, \mathrm{M}^{20}, \mathrm{~N}^{20} \\ 15 & r & 7,10 ; \mathrm{RU} & \{! p, !(\neg p \vee r)\} \mathrm{R}^{20}, \mathrm{M}^{20} \\ 16 s & 8,11 ; \mathrm{RU} & \{! q, !(\neg q \vee s)\} \mathrm{R}^{20}, \mathrm{M}^{20} \\ 17 r \vee s & 15 ; \mathrm{RU} & \{! p, !(\neg p \vee r)\} \mathrm{R}^{20} \\ 18 & r \vee s & 16 ; \mathrm{RU} & \{! q, !(\neg q \vee s)\} \mathrm{R}^{20} \\ 19 !(t \wedge \neg t) & 6 ; \mathrm{RU} & \emptyset \\ 20 ! p \vee ! q \vee !(\neg p \vee \neg q) & 1,2,3 ; \mathrm{RU} & \emptyset\end{array}$

The marking for Normal Selections is most easily checked. Only for lines 12 and 14, the disjunctions of the members of their respective conditions have been unconditionally derived in the proof. For this simple example, it is also easily seen that the formulas on lines 19 and 20 are the only minimal Dabconsequences, and hence, that all formulas that occur on lines that are not $\mathrm{N}$-marked in the proof are finally derivable from the premises according to $\mathbf{C L}_{\circ}^{n}$.

Also the marking for Reliability is easy to check. All lines that contain one or more members of $U_{20}\left(\Gamma_{2}^{\circ}\right)=\{! t \wedge \neg t, ! p, ! q, !(\neg p \vee \neg q)\}$ in their condition are R-marked.

For Minimal Abnormality, we need $\Phi_{20}\left(\Gamma_{2}^{\circ}\right)=\{\{!(t \wedge \neg t), ! p\},\{!(t \wedge \neg t), ! q\}$, $\{!(t \wedge \neg t), !(\neg p \vee \neg q)\}\}$. As an example: line 7 is M-marked, because there is no line in the proof on which $p$ is derived on a condition for which the intersection with $\{!(t \wedge \neg t), ! p\}$ (one of the members of $\Phi_{20}\left(\Gamma_{2}^{\circ}\right)$ ) is empty. Note that line 17 would be marked according to the Minimal Abnormality strategy, if line 18 would not be in the proof (and vice versa). ${ }^{8}$

\footnotetext{
${ }^{8}$ Adaptive proofs can be compared to Labelled Deductive Systems (LDSs, see [6]). The conditions that are attached to lines are comparable to the labels in LDSs: both are passed forward in subsequent derivations and both may encode additional information concerning
} 


\section{Dynamic Proofs for Explorative Reasoning}

In Section 3 we argued that the explorative reasoning style is quite natural for some application contexts. In this section, we return to the example from that section and show that the dynamic proofs of our adaptive logics are useful to explicate this kind of reasoning. The reason for this is that they allow one, in an easy way, to combine statements up to maximal consistency and to explore the consequences that follow from those combinations.

We shall present a concrete example of a dynamic proof. Notations like $! P x$ will be used as shortcuts for $(\circ P x \wedge \neg P x)$, where $1 \leq x \leq 5$, and where $P x$ stands for the corresponding statement (see P1-P5 from Section 3).

As no participant at the moment seems to outweigh one of the others, you may for the moment accept all their statements as premises:

$\begin{array}{llll}1 & \circ K & \text { PREM } & \emptyset \\ 2 & \circ L & \text { PREM } & \emptyset \\ 3 & \circ \neg(K \wedge L) & \text { PREM } & \emptyset \\ 4 & \circ((K \equiv R) \wedge \neg L) & \text { PREM } & \emptyset \\ 5 & \circ((L \equiv S) \wedge \neg K) & \text { PREM } & \emptyset\end{array}$

As you have clear preferences for hiring both Rose and Stephen, you may be interested in trying to find out whether there is a "consistent subgroup" that is in favour of hiring both these persons, and also whether some participants are against hiring them. So, your reasoning could proceed as follows:

$\begin{array}{lll}(K \equiv R) \wedge \neg L & 4 ; \mathrm{RC} & \{! P 4\} \\ K \equiv R & 6 ; \mathrm{RU} & \{! P 4\} \\ K & 1 ; \mathrm{RC} & \{! P 1\} \\ R & 7,8 ; \mathrm{RU} & \{! P 4, ! P 1\}\end{array}$

At this point, you can easily see from the condition that "Rose should be hired" follows from the statements made by $\mathrm{P} 1$ and $\mathrm{P} 4$. As you see no immediate contradictions between the statements made by $\mathrm{P} 1$ and $\mathrm{P} 4$, you may continue to derive whether there is a consistent subgroup in favour of hiring Stephen:

$\begin{array}{lll}10(L \equiv S) \wedge \neg K & 5 ; \mathrm{RC} & \{! P 5\} \\ 11 L \equiv S & 10 ; \mathrm{RU} & \{! P 5\} \\ 12 L & 2 ; \mathrm{RC} & \{! P 2\} \\ 13 S & 11,12 ; \mathrm{RU} & \{! P 5, ! P 2\}\end{array}$

The dynamic proofs also allow you to combine the statements derived on lines 9 and 13 :

the argumentative path that was used in order to derive a formula. In LDSs the term "flattening" has been used for procedures that decide which consistent set of formulas is derivable irrespective of the given labels (similarly in the context of formal argumentation, see [14]). Various flattening procedures provide different rationales how to deal with conflicting arguments. In this sense, the adaptive strategies Minimal Abnormality and Reliability and the corresponding marking definitions can be thought of as flattening procedures: given an adaptive proof with annotated proof lines that may contain various conflicts, they determine which formulas are to be deemed finally derivable. This way we gain a consequence relation that does not anymore encode the conditions on which formulas were derived. 
However, at this point you should be careful. As you can see from the conditions, you are now relying on the statements by P1, P2, P4, and P5, and there are some clear contradictions between their statements. So you better start looking for minimal disjunctions of abnormalities. Suppose the first one you derive is $! P 1 \vee ! P 5$. At that moment, you are able to continue the proof in such a way that line 14 is marked:

$\begin{array}{llll}14 & R \wedge S & 9,13 ; \mathrm{RU} & \{! P 4, ! P 1, ! P 5, ! P 2\} \mathrm{N}^{16} \\ 15 & ! P 1 \vee ! P 5 & 1,5 ; \mathrm{RU} & \emptyset \\ 16 ! P 1 \vee ! P 5 \vee ! P 2 \vee ! P 4 & 15 ; \mathrm{RU} & \emptyset\end{array}$

As you clearly will have to make change P1 or P5 their minds, you may be interested in whether they are forming consistent subgroups with other participants and what follows from those with respect to Rose and Stephen. So, for instance, you could proceed as follows

$\begin{array}{lll}17 \neg L & 6 ; \mathrm{RU} & \{! P 4\} \\ 18 \neg K & 10 ; \mathrm{RU} & \{! P 5\} \\ 19 \neg R & 18,7 ; \mathrm{RU} & \{! P 4, ! P 5\} \\ 20 \neg S & 17,11 ; \mathrm{RU} & \{! P 4, ! P 5\} \\ 21 \neg S \wedge \neg R & 19,20 ; \mathrm{RU} & \{! P 4, ! P 5\}\end{array}$

We leave it to the reader to check that line 20 will never be marked in any extension of the proof, and hence is finally derived in the proof. ${ }^{9}$

For this specific example, the strength of the dynamic proofs based on the Normal Selections strategy is that they allow one to combine different premises up to maximal consistency and to derive CL-consequences from them. They also allow one, in one and the same proof, to make inferences from different combinations, even if they are jointly inconsistent. When inferences are made that are jointly inconsistent, the conditions enable one to keep track of the premises they are derived from. Evidently, if the set of premises is inconsistent, some combinations will be inconsistent (and their CL-consequences trival), but then the Normal Selections strategy ensures that lines that contain such combinations (and all lines that are dependent on them) are marked as soon as the disjunctions of the members of their respective conditions are unconditionally derived in the proof. Finally, the adaptive proofs for the weak consequence allow one, in one and the same proof, to detect in an easy way which premises are jointly incompatible with one another (by means of the minimal Dab-formulas).

To sum up this section, the Normal Selections strategy differs from the other adaptive strategies in an important respect: lines are only marked if the abnormalities in their conditions are jointly incompatible. In the case of the RM-consequences, this immediately implies that formulas that are jointly inconsistent may be finally derived. In the above proof, all formulas on unmarked

\footnotetext{
${ }^{9}$ The only other two minimal $D a b$-formulas, besides the one on line 15 , are $! P 2 \vee ! P 4$ and $! P 1 \vee ! P 2 \vee ! P 3$.
} 
lines are finally derived (according to the Normal Selections strategy) in view of the fact that it holds for all unmarked lines that, where $\left\{! A_{1}, \ldots, ! A_{n}\right\}$ is the condition of the line, $A_{1}, \ldots, A_{n}$ are jointly compatible.

\section{In Conclusion}

In recent years, we have seen an enormous multiplication of mechanisms and logics for handling inconsistencies. Much less attention has been paid to the question for which application context which kind of logic is best suited. In this paper, we tried to address this question by distinguishing different styles of reasoning. By way of illustration, we used consequence relations that are based on the idea of maximal consistent subsets. We also showed that these consequence relations can be reconstructed in a very simple way in the framework of adaptive logics and that adaptive proofs are especially useful for the style of reasoning which is most often referred to as "credulous" and that we dubbed "explorative".

\section{APPENDIX}

In this appendix, we prove that our reconstruction of the three RM-consequence relations is adequate. We begin with some facts and lemmas concerning the relation between maximal consistent subsets and minimally abnormal models.

\section{Maximal Consistent Subsets and Minimally Abnormal Models}

A set $\Gamma$ is $\omega$-complete iff whenever $\exists \alpha A(\alpha) \in \Gamma$, then also $A(\beta) \in \Gamma$ for some constant $\beta$. $\Theta$ is said to be a maximal consistent extension of $\Gamma$ iff (i) $\Theta \supseteq \Gamma$, (ii) $\Theta$ is consistent, and (iii) for all $\Theta^{\prime} \supset \Theta, \Theta^{\prime}$ is not consistent. Let $\operatorname{MCE}(\Gamma)$ denote the set of $\omega$-complete, maximal consistent extensions of $\Gamma$.

The following are well-known facts in CL:

Fact 1 Where $\Gamma \subseteq \mathcal{W}$ is consistent: $\operatorname{MCE}(\Gamma) \neq \emptyset$.

Fact 2 Where $\Gamma \subseteq \mathcal{W}$ and $\Gamma \nvdash_{\mathbf{C L}} A$ : there is a $\Lambda \in \operatorname{MCE}(\Gamma)$ such that $A \notin \Lambda$.

Fact 3 Where $\Theta \subseteq \Gamma \subseteq \mathcal{W}$ and $\Theta$ is consistent: there is a $\Lambda \supseteq \Theta$ such that $\Lambda \in \operatorname{MCS}(\Gamma)$.

Definition 1 Where $\Gamma \subseteq \mathcal{W}$ and $\Lambda \in \operatorname{MCE}(\emptyset)$ is any $\omega$-complete, maximal consistent set of formulas in $\mathcal{W}$, we define the $\mathbf{C L}_{\circ}$-model $M_{\Lambda}^{\Gamma}=\langle D, v\rangle$ by means of $D=\{\{\beta \mid \alpha=\beta \in \Lambda\} \mid \alpha$ is a constant $\}$ and the assignment $v$ by

$$
\begin{gathered}
v(\alpha)=\{\beta \mid \alpha=\beta \in \Lambda\} \\
v(\pi)=\left\{\left\langle v\left(\alpha_{1}\right), v\left(\alpha_{2}\right), \ldots, v\left(\alpha_{n}\right)\right\rangle \mid \pi \alpha_{1} \alpha_{2} \ldots \alpha_{n} \in \Lambda\right\} \\
v(\circ A)=1 \text { iff } A \in \Gamma \\
v(p)=1 \text { iff } p \in \Lambda \text { and } p \text { is a propositional letter }
\end{gathered}
$$


Lemma 1 (i) $M_{\Lambda}^{\Gamma} \models \Lambda$, (ii) $M_{\Lambda}^{\Gamma} \models \Gamma^{\circ}$, (iii) $M_{\Lambda}^{\Gamma} \models \neg A$ for all $A \notin \Lambda$.

Proof. (i), (ii) and (iii) follow immediately by the construction above and CL-properties.

Corollary $1 A b\left(M_{\Lambda}^{\Gamma}\right)=\{\circ A \wedge \neg A \mid A \in \Gamma-\Lambda\}$.

Fact 4 Where $\Gamma \subseteq \mathcal{W}, \Lambda^{\prime} \in \operatorname{MCS}(\Gamma)$, and $\Lambda \in \operatorname{MCE}\left(\Lambda^{\prime}\right): \Gamma-\Lambda^{\prime}=\Gamma-\Lambda$.

That $\Gamma-\Lambda \subseteq \Gamma-\Lambda^{\prime}$ is immediate in view of $\Lambda^{\prime} \subseteq \Lambda$. Let $A \in \Gamma-\Lambda^{\prime}$. Since $\Lambda^{\prime} \in \operatorname{MCS}(\Gamma), \Lambda^{\prime} \cup\{A\}$ is not consistent. Thus, $A \notin \Lambda$ and hence $A \in \Gamma-\Lambda$.

Lemma 2 Where $\Lambda^{\prime} \in \operatorname{MCS}(\Gamma)$ and $\Lambda \in \operatorname{MCE}\left(\Lambda^{\prime}\right), M_{\Lambda}^{\Gamma} \in \mathcal{M}_{\mathbf{C L}_{\circ}^{m}}\left(\Gamma^{\circ}\right)$.

Proof. Suppose there is a $M \in \mathcal{M}_{\mathbf{C L}_{\circ}}\left(\Gamma^{\circ}\right)$ such that $A b(M) \subset A b\left(M_{\Lambda}^{\Gamma}\right)$. Hence, by Corollary 1 and Fact 4 there is a $A \in \Gamma-\Lambda^{\prime}$ for which $M \not \models \circ A \wedge \neg A$. Since $M \models \circ A, M \models A$. Due to the maximal consistency of $\Lambda^{\prime}$, there is a $B \in \Lambda^{\prime}$ such that $M \not \models B$ and hence $M \models \neg B$. But then $\circ B \wedge \neg B \in A b(M)-A b\left(M_{\Lambda}^{\Gamma}\right)$, - a contradiction.

Corollary 2 For each $\omega$-complete, maximal consistent extension $\Lambda$ of some maximal consistent subset of $\Gamma$ there is a minimally abnormal $\mathbf{C L}_{\circ}$-model of $\Gamma^{\circ}$ such that (i) $M \models \Lambda$, and (ii) $A b(M)=\{\circ A \wedge \neg A \mid A \in \Gamma-\Lambda\}$.

Lemma 3 For each $M \in \mathcal{M}_{\mathbf{C L}_{o}^{m}}\left(\Gamma^{\circ}\right), M=\Lambda^{\prime}$ for some $\Lambda^{\prime} \in \operatorname{MCS}(\Gamma)$.

Proof. Assume $M \in \mathcal{M}_{\mathbf{C L}_{o}^{m}}\left(\Gamma^{\circ}\right)$ and $M \not \mid \Psi$ for all $\Psi \in \operatorname{MCS}(\Gamma)$. Let $\Theta=$ $\{A|M|=A\} \cap \Gamma$. By Fact 3, there is a $\Lambda^{\prime} \in \operatorname{MCS}(\Gamma)$ such that $\Theta \subset \Lambda^{\prime}$. By Fact $1, \operatorname{MCE}\left(\Lambda^{\prime}\right) \neq \emptyset$. Take an arbitrary $\Lambda \in \operatorname{MCE}\left(\Lambda^{\prime}\right)$. Since by Corollary 1 and Fact 4 , (1) $A b\left(M_{\Lambda}^{\Gamma}\right)=\left\{\circ A \wedge \neg A \mid A \in \Gamma-\Lambda^{\prime}\right\}$, we have that (2) for any $A \in \Lambda^{\prime}-\Theta, \circ A \wedge \neg A \in A b(M)-A b\left(M_{\Lambda}^{\Gamma}\right)$ and (3) for all $A \in \Gamma-\Lambda^{\prime}, \circ A \wedge \neg A \in$ $A b(M) \cap A b\left(M_{\Lambda}^{\Gamma}\right)$. Altogether, by (1), (2), and (3), $A b(M) \supset A b\left(M_{\Lambda}^{\Gamma}\right)$, contradiction.

Lemma 4 Where $M \in \mathcal{M}_{\mathbf{C L}_{o}^{m}}\left(\Gamma^{\circ}\right), \Lambda^{\prime} \in \operatorname{MCS}(\Gamma)$ and $\Lambda \in \operatorname{MCE}\left(\Lambda^{\prime}\right), M=\Lambda^{\prime}$ iff $A b(M)=A b\left(M_{\Lambda}^{\Gamma}\right)$.

Proof. $(\Rightarrow)$ Let $M \models \Lambda^{\prime}$ for some $\Lambda^{\prime} \in \operatorname{MCS}(\Gamma)$. Since $\Lambda^{\prime} \in \operatorname{MCS}(\Gamma)$ there is no $A \in \Gamma-\Lambda^{\prime}$ such that $M \models A$. Thus, $M \models \circ A \wedge \neg A$ for all $A \in \Gamma-\Lambda^{\prime}$. Hence, since by Corollary 1 and Fact $4 A b\left(M_{\Lambda}^{\Gamma}\right)=\left\{\circ A \wedge \neg A \mid A \in \Gamma-\Lambda^{\prime}\right\}$, $A b\left(M_{\Lambda}^{\Gamma}\right) \subseteq A b(M)$. Since $M \in \mathcal{M}_{\mathbf{C L}_{o}^{m}}\left(\Gamma^{\circ}\right), A b(M)=A b\left(M_{\Lambda}^{\Gamma}\right)$.

$(\Leftarrow)$ Let $A b(M)=A b\left(M_{\Lambda}^{\Gamma}\right)$. Assume there is a $A \in \Lambda^{\prime}$ such that $M \not \models A$ and hence $M \models \neg A$. But then $\circ A \wedge \neg A \in A b(M)-A b\left(M_{\Lambda}^{\Gamma}\right)$, - a contradiction.

Corollary 3 Each minimally abnormal model $M$ of $\Gamma^{\circ}$ is such that there is a maximal consistent subset $\Lambda$ of $\Gamma$ such that (i) $M=A$ for all $A \in \Lambda$, and (ii) $A b(M)=\{\circ A \wedge \neg A \mid A \in \Gamma-\Lambda\}$.

\section{Adaptive Reconstruction of the Strong Consequences}


Theorem $1 \Gamma \vdash_{\text {Strong }} A$ iff $\Gamma^{\circ} \Vdash_{\mathbf{C L}_{o}^{m}} A$.

Proof. $(\Rightarrow)$ Let $\Lambda \vdash_{\mathbf{C L}} A$ for all $\Lambda \in \operatorname{MCS}(\Gamma)$. Let $M \in \mathcal{M}_{\mathbf{C L}_{o}^{m}}\left(\Gamma^{\circ}\right)$. By Corollary $3, M \models \Lambda$ for some $\Lambda \in \operatorname{MCS}(\Gamma)$. Hence $M=A$.

$(\Leftarrow)$ Let $\Gamma^{\circ} \Vdash_{\mathbf{C L}_{\circ}^{\mathrm{m}}} A$. Suppose there is a $\Lambda^{\prime} \in \operatorname{MCS}(\Gamma)$ such that $\Lambda^{\prime} \nvdash_{\mathbf{C L}} A$. By Fact 2 , there is a $\Lambda \in \operatorname{MCE}\left(\Lambda^{\prime}\right)$ such that $A \notin \Lambda$. By Lemma $2, M_{\Lambda}^{\Gamma} \in$ $\mathcal{M}_{\mathbf{C L}^{m}}\left(\Gamma^{\circ}\right)$ and $M_{\Lambda}^{\Gamma} \models \Lambda$ by Lemma 1 , - a contradiction.

\section{Adaptive Reconstruction of the Weak Consequences}

Recall: $\Gamma \Vdash_{\mathbf{A L}^{n}} A$ iff there is a $M \in \mathcal{M}_{\mathbf{A L}^{m}} \Gamma$ such that for all $M^{\prime} \in \mathcal{M}_{\mathbf{A L}^{m}} \Gamma$ such that $A b\left(M^{\prime}\right)=A b(M), M^{\prime}=A$.

Theorem $2 \Gamma \vdash_{\text {Weak }} A$ iff $\Gamma^{\circ} \Vdash_{\mathbf{C L}_{\circ}^{n}} A$.

Proof. $(\Rightarrow)$ Let $\Lambda^{\prime} \vdash_{\mathrm{CL}} A$ for some $\Lambda^{\prime} \in \operatorname{MCS}(\Gamma)$. Let $\Lambda \in \operatorname{MCE}\left(\Lambda^{\prime}\right)$. By

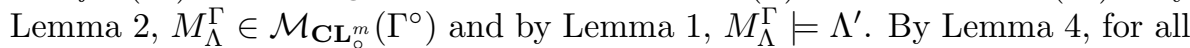
$M \in \mathcal{M}_{\mathbf{C L}_{\circ}}\left(\Gamma^{\circ}\right)$ for which $A b(M)=A b\left(M_{\Lambda}^{\Gamma}\right)$, also $M=\Lambda^{\prime}$ and thus $M \models A$. Hence, $\Gamma^{\circ} \Vdash_{\mathbf{C L}_{\circ}} A$.

$(\Leftarrow)$ Let $\Gamma^{\circ} \Vdash_{\mathbf{C L}_{\mathrm{o}}^{\mathrm{n}}} A$. Suppose for all $\Lambda^{\prime} \in \operatorname{MCS}(\Gamma), \Lambda^{\prime} \nvdash_{\mathbf{C L}} A$. Hence, by Fact 2, for each $\Lambda^{\prime} \in \operatorname{MCS}(\Gamma)$ there is a $\Lambda \in \operatorname{MCE}(\Lambda)$ such that $A \notin \Lambda$. However, for each such $\Lambda$ there is, by Corollary 2 , a $M \in \mathcal{M}_{\mathbf{C L}_{\circ}^{m}}\left(\Gamma^{\circ}\right)$ for which $M \models \Lambda$ and hence $M \not \forall A$. By Corollary 3 this means that for each $M \in \mathcal{M}_{\mathbf{C L}^{m}}\left(\Gamma^{\circ}\right)$ there is a $M^{\prime} \in \mathcal{M}_{\mathbf{C L}_{\circ}}\left(\Gamma^{\circ}\right)$ such that $A b\left(M^{\prime}\right)=A b(M)$ and $M^{\prime} \not \models A$,- contradiction.

\section{Adaptive Reconstruction of the Free Consequences}

We first present some useful meta-theory that comes with the standard format of adaptive logics.

Lemma $5 U(\Gamma)=\bigcup \Phi(\Gamma)$

Lemma $6 \Phi(\Gamma)=\left\{A b(M) \mid M \in \mathcal{M}_{\mathbf{C L}_{\circ}^{m}}(\Gamma)\right\}$.

Theorem $3 \Gamma \vdash_{\mathbf{C L}_{\circ}^{r}} A$ iff there is $a \Delta \subseteq \Omega-U(\Gamma)$ such that $\Gamma \vdash_{\mathbf{C L}_{\circ}} A \vee$ $\operatorname{Dab}(\Delta)$.

Lemma $7 \quad A \in \Gamma-\bigcap \operatorname{MCS}(\Gamma)$ iff $\circ A \wedge \neg A \in U\left(\Gamma^{\circ}\right)$

Proof. $(\Leftarrow)$ Suppose $A \notin \Gamma-\bigcap \operatorname{MCS}(\Gamma)$. Let $M$ be an arbitrary element of $\mathcal{M}_{\mathbf{C L}_{o}^{m}}\left(\Gamma^{\circ}\right)$. By Corollary $3, A b(M)=\{\circ B \wedge \neg B \mid B \in \Gamma-\Lambda\}$ for some $\Lambda \in \operatorname{MCS}(\Gamma)$. Hence, $\circ A \wedge \neg A \notin A b(M)$. Since $M$ was arbitrary in $\mathcal{M}_{\mathbf{C L}_{o}^{m}}\left(\Gamma^{\circ}\right)$, and by Lemma 6 and Lemma $5, \circ A \wedge \neg A \notin U\left(\Gamma^{\circ}\right)$.

$(\Rightarrow)$ Let $A \in \Gamma-\bigcap \operatorname{MCS}(\Gamma)$. Hence, there is a $\Lambda^{\prime} \in \operatorname{MCS}(\Gamma)$ such that $A \in$ $\Gamma-\Lambda^{\prime}$. Let $\Lambda \in \operatorname{MCE}\left(\Lambda^{\prime}\right)$. By Lemma $2, M_{\Lambda}^{\Gamma} \in \mathcal{M}_{\mathbf{C L}_{o}^{m}}\left(\Gamma^{\circ}\right)$ and by Corollary 1 and Fact $4, A b\left(M_{\Lambda}^{\Gamma}\right)=\left\{\circ B \wedge \neg B \mid B \in \Gamma-\Lambda^{\prime}\right\}$. Hence, $\circ A \wedge \neg A \in A b\left(M_{\Lambda}^{\Gamma}\right)$. By Lemma 6 and Lemma $5, \circ A \wedge \neg A \in U\left(\Gamma^{\circ}\right)$. 
Lemma 8 For each $\Lambda \in \operatorname{MCE}(\bigcap \operatorname{MCS}(\Gamma))$ there is a reliable $\mathbf{C L}_{\circ}$-model $M$ of $\Gamma^{\circ}$ such that (i) $M \models \Lambda$, and (ii) $A b(M)=\{\circ A \wedge \neg A \mid A \in \Gamma-\Lambda\}$.

Proof. Let $\Lambda \in \operatorname{MCE}(\cap \operatorname{MCS}(\Gamma))$. By Lemma $1, M_{\Lambda}^{\Gamma} \models \bigcap \operatorname{MCS}(\Gamma)$. By Corollary $1, A b\left(M_{\Lambda}^{\Gamma}\right)=\{\circ A \wedge \neg A \mid A \in \Gamma-\Lambda\} \subseteq\{\circ A \wedge \neg A \mid A \in \Gamma-\bigcap \operatorname{MCS}(\Gamma)\}$. The latter set is by Lemma 7 identical to $U\left(\Gamma^{\circ}\right)$. Hence, $M_{\Lambda}^{\Gamma} \in \mathcal{M}_{\mathbf{C L}_{\circ}^{r}}\left(\Gamma^{\circ}\right)$.

Lemma 9 Each reliable model $M$ of $\Gamma^{\circ}$ is such that $M=\bigcap \operatorname{MCS}(\Gamma)$.

Proof. Let $M \in \mathcal{M}_{\mathbf{C L}_{\circ}^{r}}\left(\Gamma^{\circ}\right)$. Assume $M \not \models \bigcap \operatorname{MCS}(\Gamma)$. Hence, there is a $A \in \bigcap \operatorname{MCS}(\Gamma)$ such that $\circ A \wedge \neg A \in A b(M)$. This is a contradiction since by Lemma $7, \circ A \wedge \neg A \notin U\left(\Gamma^{\circ}\right)$.

Theorem $4 \Gamma \vdash_{\text {Free }} A$ iff $\Gamma^{\circ} \vdash_{\mathbf{C L}_{\circ}^{r}} A$.

Proof. $(\Rightarrow)$ Let $A$ be a free consequence of $\Gamma$ and hence $\bigcap \mathrm{MCS}(\Gamma) \vdash_{\text {CL }} A$. By Lemma 9, for all $M \in \mathcal{M}_{\mathbf{C L}}\left(\Gamma^{\circ}\right), M \models \bigcap \operatorname{MCS}(\Gamma)$. Hence $\Gamma^{\circ} \Vdash_{\mathbf{C L}_{\circ}^{\mathrm{r}}} A$.

$(\Leftarrow)$ Suppose $\Gamma^{\circ} \vdash_{\mathbf{C L}^{r}} A$. Assume $\bigcap \operatorname{MCS}(\Gamma) \nvdash_{\mathbf{C L}} A$. By Fact 2 there is a $\Lambda \in \operatorname{MCE}(\bigcap \operatorname{MCS}(\Gamma))$ such that $A \notin \Lambda$. By Lemma 8 there is a $M \in \mathcal{M}_{\mathbf{C L}^{r}}\left(\Gamma^{\circ}\right)$ such that $M=\Lambda$, - a contradiction.

\section{References}

[1] Diderik Batens. Towards the unification of inconsistency handling mechanisms. Logic and Logical Philosophy, 8:5-31, 2000. Appeared 2002.

[2] Diderik Batens. A universal logic approach to adaptive logics. Logica Universalis, 1:221-242, 2007.

[3] Salem Benferhat, Didier Dubois, and Henri Prade. Some syntactic approaches to the handling of inconsistent knowledge bases: A comparative study. Part 1: The flat case. 58:17-45, 1997.

[4] Philippe Besnard and Torsten Schaub. Signed systems for paraconsistent reasoning. Journal of Automated Reasoning, 20:191-213, 1998.

[5] Phan Minh Dung. On the acceptability of arguments and its fundamental role in nonmonotonic reasoning, logic programming and n-person games. Artificial Intelligence, 77:321-358, 1995.

[6] Dov Gabbay. Labelled Deductive Systems: Principles and Applications, Vol. 1: Basic Principles. Oxford University Press, 1996.

[7] Matt Ginsberg. Essentials of Artificial Intelligence. Morgan Kaufmann Publishers Inc., San Francisco, CA, USA, 1994.

[8] John Horty. Reasons as Defaults. Oxford University Press, 2012. 
[9] John F. Horty. Some direct theories of nonmonotonic inheritance. In Dov M. Gabbay, C. J. Hogger, and J. A. Robinson, editors, Handbook of Logic in Artificial Intelligence and Logic Programming. Volume 3: Nonmonotonic Reasoning and Uncertain Reasoning. Oxford University Press, Oxford, 1994.

[10] John F. Horty. Skepticism and Floating Conclusions. Artificial Intelligence, 135:55-72, 2002.

[11] David Makinson. General patterns in nonmonotonic reasoning. In Dov M. Gabbay, C.J. Hogger, and J. A. Robinson, editors, Handbook of Logic in Artificial Intelligence and Logic Programming, volume 3, pages 35-110. Oxford University Press, Inc., 1994.

[12] David Makinson and Karl Schlechta. Floating conclusions and zombie paths: two deep difficulties in the "directly skeptical" approach to defeasible inheritance nets. 48(2):199-209, 1991.

[13] David Makinson and Leendert van der Torre. Constraints for Input/Output logics. Journal of Philosophical Logic, 30(2):155-185, April 2001.

[14] Simon Parsons. On precise and correct qualitative probabilistic inference. International Journal of Approximate Reasoning, 35(2):111-135, 2004.

[15] John Pollock. Cognitive Carpentry. Bradford/MIT Press, 1995.

[16] Henry Prakken. Combining sceptical epistemic reasoning with credulous practical reasoning. In P.E. Dunne and T.J.M. Bench-Capon, editors, Computational Models of Argument. Proceedings of COMMA-06., pages 311-322, Amsterdam, 2006. IOS Press.

[17] Henry Prakken. An abstract framework for argumentation with structured arguments. 1(2):93-124, 2011.

[18] Nicholas Rescher and Ruth Manor. On inference from inconsistent premises. Theory and Decision, 1:179-217, 1970.

[19] Christian Straßer. Adaptive Logics and Defeasible Reasoning. Applications in Argumentation, Normative Reasoning and Default Reasoning. Springer, in print.

[20] Christian Straßer and Joke Meheus. Simple representations of some adaptive consequence relations in a Kripke frame. Forthcoming.

[21] David S. Touretzky, John F. Horty, and Richmond H. Thomas. A clash of intuitions: The current state of nonmonotonic multiple inheritance systems. In IJCAI-8\%, pages 476-482. Morgan Kaufmann, 1987. 